SPACE RESEARCH COORDINATION CENTER

\title{
TRANSFER OF EXCITATION ENERGY FROM NITROGEN MOLECULES TO SODIUM ATOMS
}

\author{
1 " 1 BY \\ J. E. MENTALL, H. F. KRAUSE AND W. L. FITE \\ DEPARTMENT OF PHYSICS
}

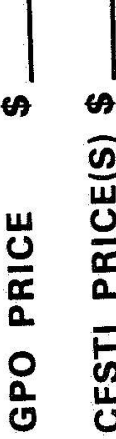

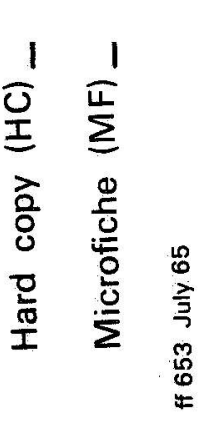

29 SRCC REPORT NO. 46 象

UNIVERSITY OF PITTSBURGH

PITTSBURGH, PENNSYLVANIA

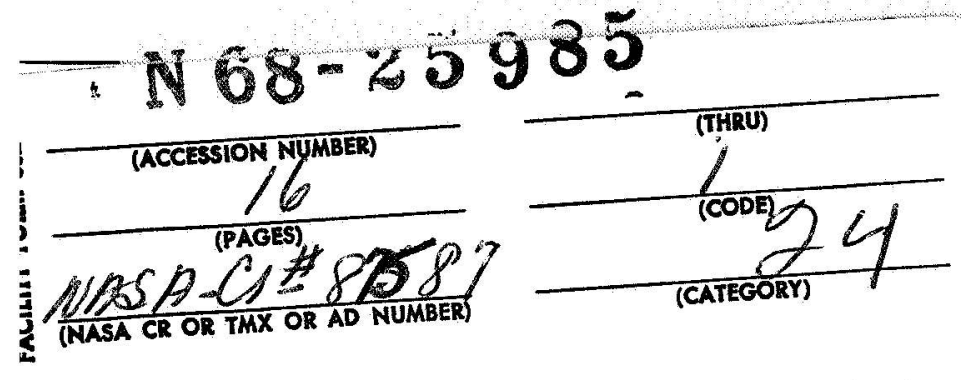

MAY 1967/() 
3 Transfer of Excitation Energy from Nitrogen Molecules to Sodium Atoms*

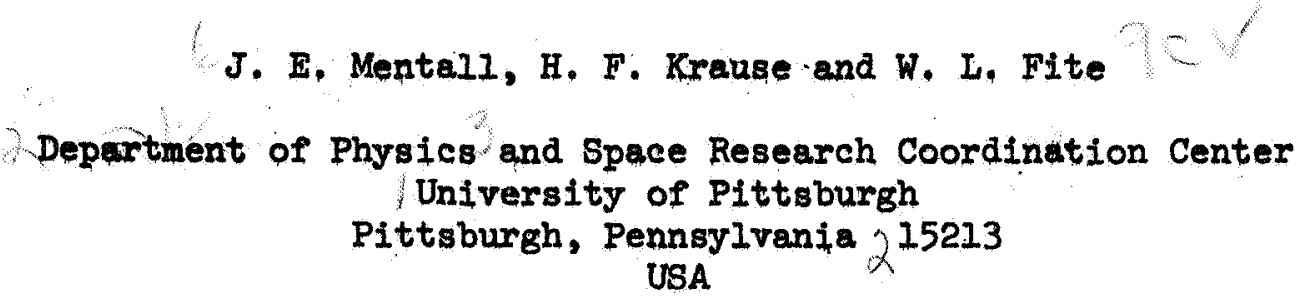

For presentation at the Faraday Society's General Discussion on Molecular Dymanics of the Chemical Reactions in Gases, Toronto University, September, 1967.

"This research was supported in part by the National Aeronautics and Space Administration and in part by the Advanced Research Projects Agency. 
Transfer of Excitation Energy from Nitrogen Molecules to Sodium Atoms

J. E. Mentall, H. F. Krause and W. L. Fite

Department of Physics and Space Research Coordination Center

University of Pittsburgh

Pittsburgh, Pennsylvania 15213

USA

ABSTRACT

Bxperiments have been performed in which a beam of $\mathrm{N}_{2}$, issuing from a furnace source operating at temperatures from 2000 to $3000^{\circ} \mathrm{K}$, crossed a beam of sodium atoms. : Sodium D-Iine radiation was observed as a product of the collisions of the two beams. The radiation appears to occur primarily from the transfer of internal energy in the diatomic molecule, which because of the nature of the molecules must be vibrational and rotational only, into electronic energy in the sodium, presumably through the formation of a transitory $\mathrm{NaN}_{2}$ * complex. On the assumption that only internal energy transfer is involved, rate coefficients of approximately $10^{-10} \mathrm{~cm}^{3} / \mathrm{sec}$ are found for those molecules with sufficient internal energy to excite the sodium. Weak temperature dependences of the observed rate coefficients are discussed. 
The quenching of sodium resonance radiation by diatomic molecules is wellknown and has been studied extensively under a variety of laboratory conditions. ${ }^{1}$ In the case of $\mathrm{N}_{2}$ as the quenching gas, the first electronic state is more than $6 \mathrm{eV}$ above the groundstate and the transfer must therefore involve the conversion of $2.10 \mathrm{eV}$ of electronic energy in the $\mathrm{Na}$ into vibrational and rotational energy in the $\mathbb{N}_{2}$ and kinetic energy. The effective cross sections are large, typlcal values published ranging from 6 to $40 \times 10^{-16} \mathrm{~cm}^{2}$. Because one can find vibrational excitation levels which are nearly resonant with the energy of the sodium excitation, it is often assumed that quenching occurs primarily through the excitation of vibrational energy in the $\mathrm{N}_{2}$. This presumably must proceed through the formation of the $\mathrm{NaN}_{2}^{*}$ complex, which might, however, give products with low internal energy and large kinetic energies.

Whether the excitation is transferred with little involvement of kinetic energy is of considerable interest to the understanding of certain types of aurore in which very strong sodium radiation is observed. D. M. Hunten ${ }^{2}$ has considered the auroral sodium excitation problem in detail and has concluded that direct excitation of the sodium by energetic electrons is untenable; the competition for these electrons is overwhelmingly won at the auroral altitudes by the much more abundant $\mathrm{N}_{2}$ molecules, which become vibrationally excited to the $v=8$ state with large cross section. ${ }^{3}$ Hunten proposed that the excitation of the sodium arises through transfer of this vibrational energy in the $\mathrm{N}_{2}$ to electronic excitation in the Na, a process which has been observed to occur 4 and for which a lower limit to the cross section has come from shock tube data. ${ }^{5}$ Whether this proposal is tenable depends on the cross section for such a transfer process. Detailed balancing arguments applied to the Na quenching cross sections are not fully satisfactory in the absence of a guarantee that quenching does not result in products with low internal excitation and large kinetic energies. A direct quantitative experiment on transfer of energy from $\mathrm{N}_{2}$ to $\mathrm{Na}$ is clearly called for, This paper describes such an experiment. 
EXPERIMENTAL

The experiment, which utilizes beams crossed at $90^{\circ}$ is shown schematically in Fig. 1. The diatomic gas is excited by heating in tungsten furnace operating between 2000 and $3000^{\circ} \mathrm{K}$. Since the furnace geometry is such that each molecule would make on the average approximately $10^{3}$ collisions with the walls before escaping into the beam and the use of tungsten wire wadding inside the furnace further increases contact of the gas with the hot surfaces of the furnace, it is assumed that the gas reaches equilibrium with the furnace walls and the degree of excitation of internal energy states is that given by the Boltzmann distribution. Typically, therefore, only about one molecule in $10^{4}$ has sufficient internal energy to excite the $2.10 \mathrm{eV}$ sodium excited state. The slow relaxation rate for vibrational excitation in $\mathrm{N}_{2}$ implies that the vibrational population remains very nearly that in the furnace even after the gas emerges and forms the molecular beam.

The sodium atom beam is produced in a double-chamber oven, the temperature of the rear chamber governing the beam intensity and the temperature of the front chamber determining the beam speed.

Sodium D-line photons produced by the interaction of the $\mathrm{N}_{2}$ and $\mathrm{Na}$ beams are detected with an EMI 9558A photomultiplier which views the interaction region. Wavelength discrimination is achieved using $10 \AA$ bandpass interference filter preceded by a collimating lens. This bandwidth still admits a large signal due to radiation from the hot furnace reflecting from the blackened walls of the vacuum chamber. As a matter of convenience in separating such reflected radiation from radiation arising from the intersecting beams, the sodium beam is modulated at $1440^{\circ} \mathrm{cps}$ by a rotating chopper wheel. Under these circumstances the desired signal is identified by its occurring at the sodium beam modulation frequency and in specified phase. The signal from the photomultiplier is fed 
to a cathode follower and a condenser blocks out the d.c. signal from the reflected radiation. The remaining a.c. signal is passed through a narrowband-amplifier and then to a phase-sensitive detector and integrator, output is displayed on a pen recorder.

Modulated signals in addition to those from the interaction of the excited $\mathrm{N}_{2}$ molecules with the $\mathrm{Na}$ atoms are obtained by resonant scattering of the thermal light from the furnace. By making the collimation sufficiently tight that about $90 \%$ of the light intersecting the Na beam comes from the hole in the furnace, the light can to good approximation be taken as conforming to the Planck black-body radiation formula. Since the resonant scattering cross section for D-line radiation is well known, the experiment can therefore proceed by comparing the scattered black-body-light signal to the signal produced by the particle interaction, both detected by the same optical system. Effectively, black-body radiation scattering by sodium atoms calibrates in one step both the intensity of the $\mathrm{Na}$ beam and the characteristics of the optical system.

In practice the situation is slightly more complex being so rendered by the very large intensity of the resonantly scattered black-body-light compared to the light from the particle interaction. In order to detect the particle interaction signals it is necessary to attenuate the black-body-light by about three orders of magnitude. This is accomplished by the insertion of the absorption cell containing sodium vapor shown in Fig. 1 immediately before the sodium cross beam. Operating at a temperature of about $490^{\circ} \mathrm{K}$, the vapor pressure in this cell is about $5 \times 10^{-4}$ torr. Because the cross section for resonant absorption is approximately $10^{-11} \mathrm{~cm}^{2}$, this pressure is adequate to provide the needed attenuation. At the same time, a pressure of $5 \times 10^{-4}$ torr affects only approximately $1 \%$ of the $\mathrm{N}_{2}$ molecules passing through the cell.

The experimental procedure is as follows. First the sodium oven temperatures are set and the sodium beam established. A hot wire surface ionization detector 
(not shown in Fig. 1) indicates the presence of an adequately intense sodium beam - Second, the tungsten oven is turned on and its temperature measured using an optical pyrometer looking into the furnace aperture. The signal from the resonantly scattered furnace light is noted.

The sodium vapor absorption cell is then heated to its temperature and the scattered light signal drops. Gas is then admitted to the furnace and the rise of signel of sodium light is noted.

Lastly, the change in pressure in the final vacuum chamber on turning on and off the molecular beam is noted. From the pumping speed for the molecular beam gas (abtained from a separate measurement of the pressure time constant of the system) and the pressure change on admission of the beam, the current carried by the molecular beam is obtained.

Since the attenuation of the sodium cell is known and is kept constant, the furnace temperature can be changed and the signals before and after addition of the molecular gas to the furnace can be noted, giving data at a second. temperature.

From the measured geometry of the apparatus, the measured light signals and the measured molecular beam current, and using the known sodium resonant scattering cross section and Planck's distribution law for the intensity of radiation resonantly scattered by the sodium beam, a rate coefficient is obtained. The rate so obtained is appropriate to the assumption that the light produced in the particle collisions is isotropically distributed. (The angular distribution of the resonantly scattered light is only $2 \%$ away from being isotropic ${ }^{6}$ and this correction was not made in treating the data.) 


\section{RESULTS}

The direct output of the experiment is a rate coefficient defined in the usual way,

$$
S=\alpha n_{1} n_{2}
$$

where $S$ is the total signel per unit time and volume, $n_{1}$ and $n_{2}$ are the total number densities of the sodium atoms and the diatomic gas molecules, respectively, and $\alpha$ is the rate coefficient. When the two beams have Maxwell-Boltzmann velocity distributions, the rate constant is given by

$$
\alpha=\frac{2\left(\beta_{1} \beta_{2}\right)^{\frac{3}{2}}}{\delta} \int_{0}^{\infty} v^{4}\left\{\frac{\int_{0}^{\infty} Q(\varepsilon, v) g(\varepsilon) e^{-\frac{\varepsilon}{k T_{2}}} d \varepsilon}{\int_{0}^{\infty} g(\varepsilon) e^{-\frac{\varepsilon}{k T_{2}}} d \varepsilon}\right\} I_{1}\left(\delta v^{2}\right) e^{-\gamma v^{2}} d v
$$

where $B_{i}=\frac{m_{i}}{2 k T_{i}}, \quad \gamma=\frac{2}{2}\left(B_{1}+\beta_{2}\right), \quad \delta=\frac{1}{2}\left(B_{1}-\beta_{2}\right), \quad v$ is the relative velocity in the center of mass, and $I_{1}(x)$ is the first order modified Bessel's function of the first kind. In the quantity in brackets in the integrand $Q(\varepsilon, v)$ is the cross section for the energy transfer which depends in general on both the relative velocity and the energy of internal excitation, $\varepsilon . g(\varepsilon)$ is the statistical weight density of states of internal energy $\varepsilon$. Since the population of internal energy states depends only on the temperature of the molecular beam furnace source, $\mathrm{T}_{2}$, this temperature appears in the-Boltzmann factor. The total quantity in brackets is, of course, the reaction cross section for a given relative velocity averaged over all internal energy states thermally populated.

It is convenient to re-write (2) in terms not of the relative velocity, $v$, but of kinetic energy in the center of mass system, $E=\frac{1}{2} \mu v^{2}$, where $\mu$ is the reduced mass. The expression becomes 


$$
\alpha=\frac{\left(\beta_{1} \beta_{2}\right)^{\frac{3}{2}}}{\delta}\left(\frac{2}{\mu}\right)^{\frac{5}{2}} \int_{0}^{\infty} E^{\frac{3}{2}}\left\{\frac{\int^{Q} Q(\varepsilon, E) g(\varepsilon) \epsilon^{-\frac{\varepsilon}{k T}} d \varepsilon}{\int_{0}^{\infty} g(\varepsilon) e^{-\frac{\varepsilon}{k T_{2}}} d \varepsilon}\right\} I_{1}\left(\frac{2 \delta E}{\mu}\right) e^{-\frac{2 \gamma E}{\mu}} d E
$$

Conservation of energy requires of course that $Q(\varepsilon, E)$ vanish for $\varepsilon+E<E_{0}$, the threshold energy for excitation of the sodium (2.10 ev.)

Since $\alpha$ itself is strongly dependent on the temperature $T_{2}$ through the Boltzmann factor, it is convenient to introduce a second quantity, $n$, given by

$$
n=\alpha e^{\frac{E_{0}}{k T_{2}}}
$$

The quantity $n$ is plotted as a function of $\mathbb{T}_{2}$ in Fig. 2. The most striking points to note are the weak dependence of $n$ on temperature and the large magnitudes.

In the absence of more detailed information than that provided by the thermal beam experiments, the physical interpretation of the results must necessarily be semi-quantitative. Nonetheless some consideration of the results reveal the main essentials of the basic processes involved. These considerations are based upon the extent to which the excitation of the sodium depends on the kinetic energy as opposed to internal energy in the molecule.

It is of interest to consider two extreme cases, the first assuming that internal energy is irrelevant and all the effect comes from the kinetic energy of the molecules. In this case; the term in brackets in Eq. (3) becomes simply Q(E) so that

$$
\eta=\frac{\left(B_{1} B_{2}\right)^{\frac{3}{2}}}{\delta}\left(\frac{2}{\mu}\right)^{\frac{5}{2}} e^{\frac{E_{0}}{k T_{2}}} \int_{E_{0}}^{\infty} E_{Q}^{\frac{3}{2}} Q(E) I_{1}\left(\frac{2 \delta E}{\mu}\right) e^{-\frac{2 \gamma E}{\mu}} d E,
$$

where $E_{0}=2.10 \mathrm{eV}$. Under conditions of the experiment the value of $\frac{2 \delta E_{0}}{\mu}$ is 
about 20 , so that the asymptotic form of the Bessel function can be used, and the expression simplifies to

$$
\eta=\left(\frac{\beta_{1} \beta_{2}}{\delta}\right)^{\frac{3}{2}} \frac{4}{\sqrt{2 \pi}} \frac{1}{\mu^{2}} \int_{E_{0}}^{\infty} E Q(E) e^{-\frac{1}{k T_{2}}\left(\frac{m_{2}}{\mu} E-E_{0}\right)} d E .
$$

Since the minimum value of $E$ is $E_{0}$ and $m_{2}>\mu, \frac{m_{2}}{\mu} E-E_{0}$ must always be positive. For nitrogen where the ratio of the mass to the reduced mass is about 2.2 the exponential in the integral must give a temperature dependence for $n$ at least as strong as

$$
e^{-\frac{1.2 \mathrm{E}_{0}}{\mathrm{kT}_{2}}}
$$

This would require a factor in excess of 10 difference in the values of $n$ at 2200 and $2800^{\circ} \mathrm{K}$. Fig. 2 shows a much weaker temperature dependence, from which we can conclude that kinetic energy cannot be the major cause of the sodium excitation.

It can also be shown that the cross sections for those molecules having sufficient kinetic energy to produce the observed signals would have to be of the order of $10^{-10} \mathrm{~cm}^{2}$ which is unreasonably high.

Turning to the other extreme, if it is assumed that kinetic energy plays no role whatever, then $Q(\varepsilon, E)=Q(\varepsilon)$ and the expression for $n$ reduces to

$$
n=\bar{v} \frac{\int_{0}^{\infty} Q\left(E_{0}+\varepsilon\right) g\left(E_{0}+\varepsilon\right) e^{-\frac{\varepsilon}{k T_{2}}} d \varepsilon}{\int_{0}^{\infty} g(\varepsilon) e^{-\frac{\varepsilon}{k T_{2}}} d \varepsilon}
$$

Since for the molecules of interest here only vibrational and rotational states are available, the cross section for the $n$-th vibrational state and rotational energy, $u$, can be written $Q_{n}(u)$. The rotational weight density, $1 / B$, where $B$ is the rotational constant, and the vibrational weight density $\Sigma \delta\left(\varepsilon-n h \omega_{0}\right)$ 
(using the simple harmonic oscillator approximation) can be inserted to yield

$$
n=\bar{v}_{k}\left(1-e^{\left.-\frac{h \omega_{0}}{k T_{2}}\right)_{e}-\frac{\left(n_{0} h \omega_{0}-E_{0}\right)}{k T_{2}}}\left(\bar{Q}_{n_{0}}+\bar{Q}_{n_{0}+1} e^{-\frac{h \omega_{0}}{k T_{2}}}+\bar{Q}_{n_{0}+2^{e}} e^{-\frac{2 h \omega_{0}}{k T_{2}}}+\ldots\right)\right.
$$

where $n_{0}$ is the quantum number of the lowest vibrational state whose energy exceeds $E_{0}, \bar{v}$ is the average relative velocity and,

$$
\bar{Q}_{n}=\frac{1}{k T_{2}} \int_{0}^{\infty} Q_{n}(u) e^{-\frac{u}{k T_{2}}} d u,
$$

is the cross section for a given vibrational level averaged over the rotational levels.

Now, $\bar{v}$ varies by less than 10\% over the temperature range of the experiments and for $n_{0}=8$ the energy difference in the third factor of Eq. (7) is but $0.11 \mathrm{eV}$ giving a weak temperature dependence. Further, while the third factor weakly increases with temperature the second term decreases with temperature such that the product of the two is very nearly temperature-independent. Thus any appreciable temperature dependence must arise from the series. The temperature dependence in Fig. 2 can be quite consistent with the assumption of internal energy counting for the entire energy transfer. In the absence of detailed information about the cross sections, $\bar{Q}_{n}$, averaged over the rotational energy states, little more can be said, except to note that the increase of $\bar{Q}_{n}$ with temperature offers the possibility that the cross sections for transfer from vibrational states of $\mathrm{n}>8$, may be quite large.

In this extreme case, where only internal energy is considered important, $\eta$ takes on the physical significance of being the reaction rate constant for those molecules with sufficient energy to cause the excitation of the sodium. One can then deduce that for these molecules the effective cross section for the excitation is about $2 \times 10^{-15} \mathrm{~cm}^{2}$, which is of the correct magnitude to 
explain the auroral phenomena. The number also exceeds the lower limit of $10^{-15} \mathrm{~cm}^{2}$ given by Hurle based on shock tube data. 5

One further exercise was performed on the data of this experiment. Detalled balancing was examined for the sodium excitation and sodium quenching cross sections. The sodium excitation cross section was taken to be the rate coefficient measured in this experiment simply divided by the mean relative velocity of the molecules and detailed balancing was used to derive quenching cross sections of differing final kinetic energy and vibrational energy of the $\mathbb{N}_{2}$ molecule. It was found that in order to obtain quenching cross sections within the range of experimental values which have been reported, it appears necessary to have the molecule left in vibrational states of at least $n=6$. Although some bias is injected into this argument in the manner in which an excitation cross section is obtained, we regard the consistency obtained as supporting the belief that internal energy is more important than kinetic energy in the interactions leading either to excitation or to quenching of sodium atoms in collisions with nitrogen molecules. 


\section{FIGURE CAPTIONS}

1. Sketch of experimental arrangement. For discussion see text. The figure does not show the hot-wire detector for the sodium beam nor the light baffles used to cut down scattered light originating from the tungsten furnace.

2. Plot of $n$ vs. $\mathbb{T}_{2}$, the temperature of furnace from which the $\mathrm{N}_{2}$ beam issues. $n$ can be thought of crudely as a rate coefficient for those nitrogen molecules with sufficient energy to effect the excitation of the sodium. 
REFERENCES

1. D. R. Jenkins, Proc. Roy. Soc., 1966, A293, 493.

2. D. M. Hunter, J. Atm. Terr. Physics, 1965, 27, 538.

3. G. J. Schulz, Phys. Rev., 1962, 125, 229.

4. W. L. Starr, J. Chem. Phys , , 1965, 43, 73.

5. I. R. Hurle, J. Chem. Phys., 1964, 41, 3911.

6. J. W. Chamberlain, Physics of the Aurora and Airglow (Academic Press, New York, 1961), p. 448. 


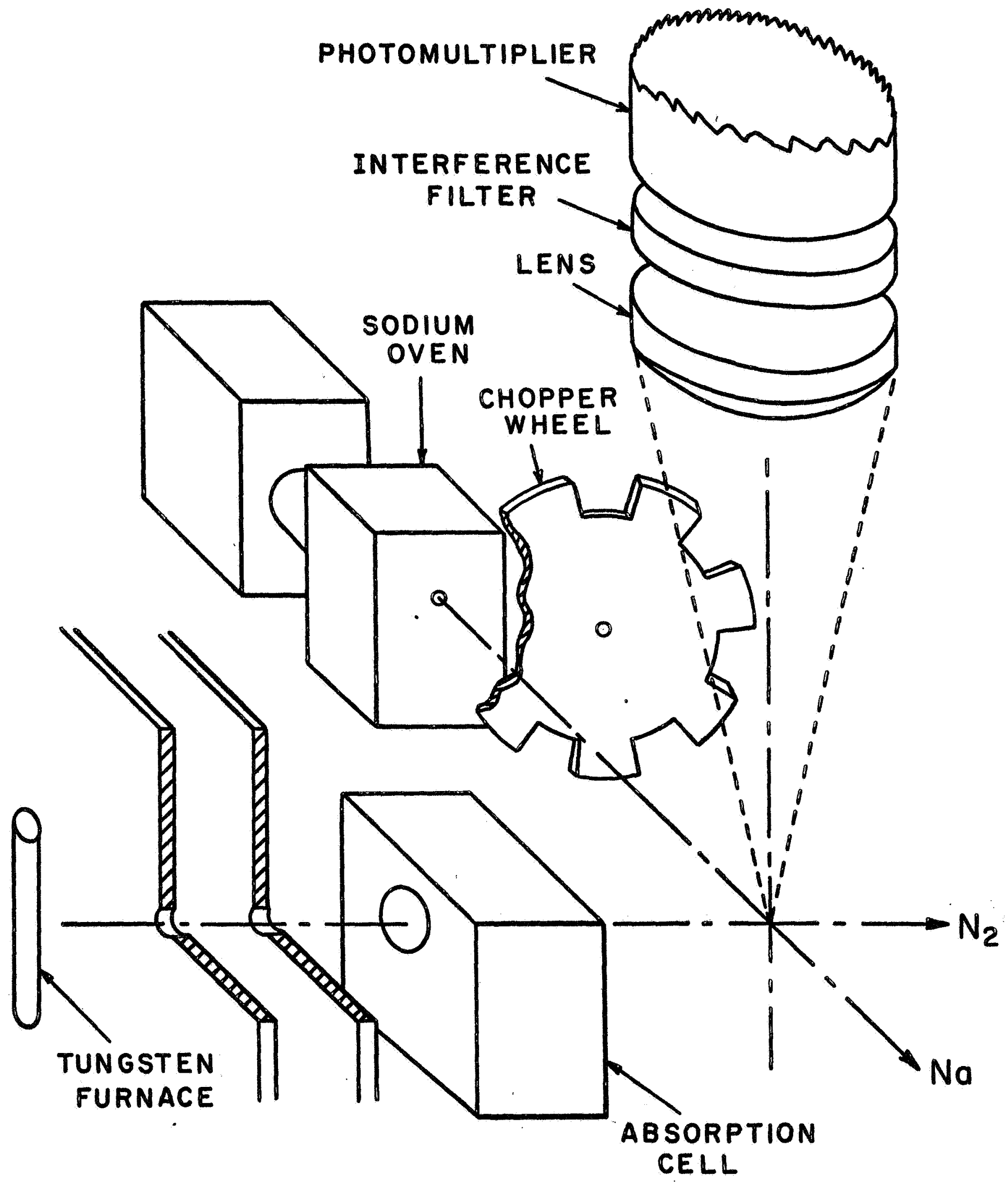




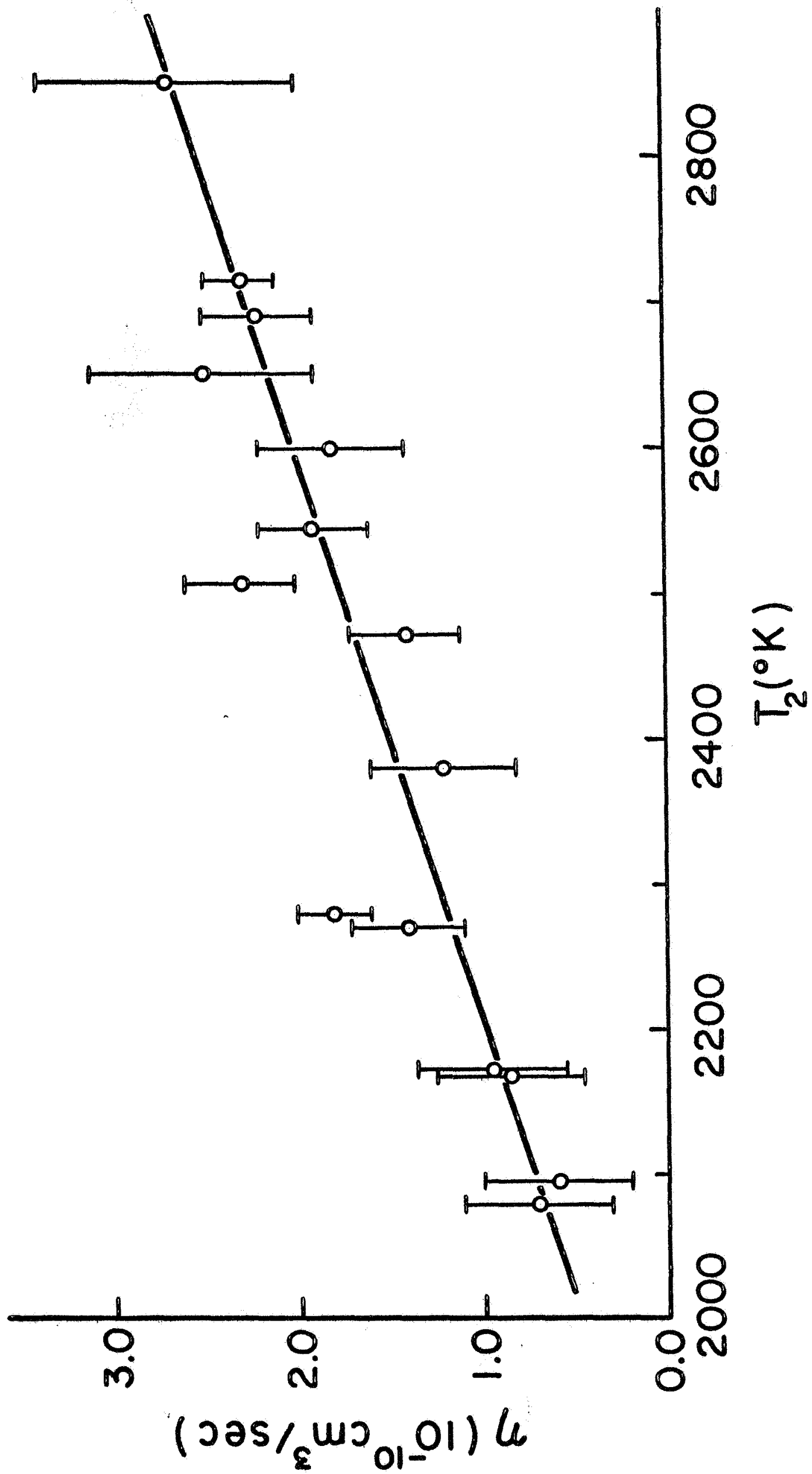

